Ferrata Storti Foundation

\title{
Pharmacological modulation of CXCR4 cooperates with BET bromodomain inhibition in diffuse large B-cell lymphoma
}

\author{
Clara Recasens-Zorzo, ${ }^{1}$ Teresa Cardesa-Salzmann, ${ }^{2}$ Paolo Petazzi, ${ }^{3}$ \\ Laia Ros-Blanco, ${ }^{4}$ Anna Esteve-Arenys, ${ }^{1}$ Guillem Clot, ${ }^{1}$ \\ Martina Guerrero-Hernández, ${ }^{1}$ Vanina Rodríguez, ${ }^{1}$ Davide Soldini, ${ }^{2}$ \\ Alexandra Valera, ${ }^{2}$ Alexandra Moros, ${ }^{1}$ Fina Climent, ${ }^{5}$ Eva González-Barca, ${ }^{6}$ \\ Santiago Mercadal, ${ }^{6}$ Leonor Arenillas, ${ }^{7}$ Xavier Calvo, ${ }^{7}$ José Luís Mate, ${ }^{8}$ \\ Gonzalo Gutiérrez-García, ${ }^{9}$ Isolda Casanova, ${ }^{3,10}$ Ramón Mangues, ${ }^{3,10}$ \\ Alejandra Sanjuan-Pla, ${ }^{11}$ Clara Bueno, ${ }^{3}$ Pablo Menéndez,, 12 \\ Antonio Martínez, ${ }^{1,2}$ Dolors Colomer, ${ }^{1,2}$ Roger Estrada Tejedor, ${ }^{4}$ Jordi Teixidó, ${ }^{4}$ \\ Elias Campo, ${ }^{1,2}$ Armando López-Guillermo, ${ }^{1,9}$ José Ignacio Borrell, ${ }^{4}$ \\ Luis Colomo, ${ }^{2,7}$ Patricia Pérez-Galán ${ }^{1}$ and Gaël Roué ê,13 $^{1,13}$
}

Haematologica 2019

Volume 104(4):778-788

\begin{abstract}
${ }^{1}$ Division of Hemato-Oncology, Institut d'Investigacions Biomediques August Pi I Sunyer (IDIBAPS), CIBERONC, Barcelona; ${ }^{2} \mathrm{Hematopathology} \mathrm{Unit,} \mathrm{Department} \mathrm{of} \mathrm{Pathology,}$ Hospital Clinic, Barcelona; ${ }^{3}$ Josep Carreras Leukemia Research Institute, Department of Biomedicine, School of Medicine, University of Barcelona; ${ }^{4}$ Grup d'Enginyeria Molecular, IQS School of Engineering, Universitat Ramon Llull, Barcelona; ${ }^{5}$ Pathology Department, Hospital Universitari de Bellvitge, L'Hospitalet de Llobregat; 'Institut Catalá d'Oncología, Hospital Duran I Reynals, L'Hospitalet de Llobregat; 'Pathology Department, IMIM, Hospital del Mar, Barcelona; ${ }^{8}$ Pathology Department, Hospital Universitari Germans Trias i Pujol, Badalona; ${ }^{9}$ Department of Hematology, Hospital Clinic, Barcelona; ${ }^{10} \mathrm{Grup}$ d'Oncogènesi i Antitumorals, Institut d'Investigacions Biomèdiques Sant Pau (IIB-Sant Pau) and Centro de Investigación Biomédica en Red CIBER-BBN, Barcelona;

${ }^{11} \mathrm{Hematology}$ Research Group, Health Research Institute La Fe, Valencia; ${ }^{12}$ Institucio Catalana de Recerca I Estudis Avançats (ICREA), CIBERONC, Barcelona and ${ }^{13}$ Laboratory of Experimental Hematology, Department of Hematology, Vall d'Hebron Institute of Oncology, Vall d'Hebron University Hospital, Barcelona, Spain
\end{abstract}

LC, PP-G and GR. share senior authorship.

\section{Correspondence:}

GAËL ROUÉ

groue@vhebron.net

PATRICIA PÉREZ-GALÁN

pperez@clinic.ub.es

Received: September 8, 2017.

Accepted: June 25, 2018.

Pre-published: June 28, 2018.

doi:10.3324/haematol.2017.180505

Check the online version for the most updated information on this article, online supplements, and information on authorship \& disclosures: www.haematologica.org/content/104/4/778

(C)2019 Ferrata Storti Foundation

Material published in Haematologica is covered by copyright. All rights are reserved to the Ferrata Storti Foundation. Use of published material is allowed under the following terms and conditions:

https://creativecommons.org/licenses/by-nc/4.0/legalcode. Copies of published material are allowed for personal or internal use. Sharing published material for non-commercial purposes is subject to the following conditions:

https://creativecommons.org/licenses/by-nc/4.0/legalcode, sect. 3. Reproducing and sharing published material for commercial purposes is not allowed without permission in writing from the publisher.

\section{ABSTRACT}

onstitutive activation of the chemokine receptor CXCR4 has been associated with tumor progression, invasion, and chemotherapy resistance in different cancer subtypes. Although the CXCR4 pathway has recently been suggested as an adverse prognostic marker in diffuse large B-cell lymphoma, its biological relevance in this disease remains underexplored. In a homogeneous set of 52 biopsies from patients, an antibody-based cytokine array showed that tissue levels of CXCL12 correlated with high microvessel density and bone marrow involvement at diagnosis, supporting a role for the CXCL12-CXCR4 axis in disease progression. We then identified the tetra-amine IOS01.01RS as a potent inverse agonist of the receptor, preventing CXCL12mediated chemotaxis and triggering apoptosis in a panel of 18 cell lines and primary cultures, with superior mobilizing properties in vivo than those of the standard agent. IOS-01.01RS activity was associated with downregulation of $\mathrm{p}$-AKT, p-ERK1/2 and destabilization of MYC, allowing a synergistic interaction with the bromodomain and extra-terminal domain inhibitor, CPI203. In a xenotransplant model of diffuse large Bcell lymphoma, the combination of IOS-01.01RS and CPI203 decreased tumor burden through MYC and p-AKT downregulation, and enhanced the induction of apoptosis. Thus, our results point out an emerging role of CXCL12-CXCR4 in the pathogenesis of diffuse large B-cell lymphoma and support the simultaneous targeting of CXCR4 and bromodomain proteins as a promising, rationale-based strategy for the treatment of this disease. 


\section{Introduction}

Diffuse large B-cell lymphoma (DLBCL) is the most common type of non-Hodgkin lymphoma among adults, accounting for $30-40 \%$ of newly diagnosed cases. ${ }^{1}$ Although the introduction of rituximab into clinical practice has increased the survival of affected patients by 10$15 \%,{ }^{2} 60 \%$ of patients with high-risk DLBCL are still not cured by immunochemotherapy and have a dismal outcome. For this subgroup, the development of more effective salvage strategies remains an important objective.

Gene expression profiling studies have confirmed the heterogeneity of DLBCL, not otherwise specified, and have recognized two major subtypes according to the putative cell of origin, i.e. activated $\mathrm{B}$-cell $(\mathrm{ABC})$ and germinal center B-cell (GCB). ${ }^{3}$ These studies have also evidenced the role of the stromal microenvironment in the pathogenesis of the disease, as well as in environmentmediated resistance of DLBCL cells to chemotherapeutics. ${ }^{4}$ As normal B cells are strongly dependent on soluble cytokines for their development and throughout their whole lifespan, it is not surprising that malignant B cells exploit their microenvironment interaction properties for their own selective advantage. ${ }^{5}$ The CXCR4 chemokine receptor (fusin, CD184) has a well-known function in normal B-cell development, including homing of hematopoietic stem cells to the bone marrow, B-cell and T-cell lymphopoiesis, leukocyte trafficking, and B-cell positioning in the germinal center, among others. ${ }^{6-11}$ CXCR4 overexpression has been linked to metastasis in a variety of cancers and recently identified as an adverse prognostic factor in DLBCL. ${ }^{12,13}$ Accordingly, the CXCR4 ligand, CXCL12 (SDF-1 $\alpha$ ), is among the genes included in the proangiogenic "stromal 2 gene signature" associated with an unfavorable outcome in DLBCL. ${ }^{4}$ This cytokine is secreted by normal and tumor stroma and is a major regulator of cell chemotaxis. ${ }^{14}$ Leukemia stem cells and other CXCR4-expressing tumors utilize the CXCL12-CXCR4 signaling axis to localize to vascular and endosteal niches normally restricted to hematopoietic stem cells, ${ }^{15}$ thus obtaining protection from the effects of cytotoxic chemotherapy and making these niches look like a reservoir for minimal residual disease and relapses. ${ }^{16-18}$ CXCR4 expression allows tumor cell migration, and homing of the neoplastic cells to sites where nonmalignant stromal cells express CXCL12. ${ }^{15}$ This latter promotes tumor progression by recruiting CD31+ endothelial progenitor cells and consequent tumor angiogenesis. ${ }^{19-21}$ CXCR4 is expressed in hematologic tumors as diverse as B-cell acute lymphoblastic leukemia, acute myeloid leukemia, chronic lymphocytic leukemia and multiple myeloma, and various ongoing clinical trials for patients with relapsed/refractory hematologic malignancies and recurrent high-grade glioma are evaluating the benefit of targeting the tumor microenvironment through CXCL12-CXCR4. $22-27$

Here we analyzed the clinical significance of CXCL12 expression level in a homogeneous series of patients with de novo DLBCL. We further characterized a new, potent CXCR4 inhibitor showing in vitro and in vivo combinational activity with a BET bromodomain inhibitor, thereby demonstrating that dual targeting of CXCR4 and MYC represents a promising therapeutic strategy for DLBCL.

\section{Methods}

\section{Patients' samples}

Fifty-two biopsy specimens from untreated patients with de novo DLBCL from the Catalan lymphoma-study group (GELCAB) were included in this study (see details in Online Supplementary Table S1 and the Online Supplementary Materials and Methods). For functional studies, primary tumor cells from five patients were isolated, cryopreserved, and conserved within the hematopathology collection of our institution (Hospital Clínic-IDIBAPS Biobank R121001-094), as previously described. ${ }^{28}$ Primary cultures were maintained in complete medium in the presence of the mesenchymal cell line StromaNKTert (Riken BioResource Center) ${ }^{29}$ at a 4:1 ratio, to prevent spontaneous ex vivo apoptosis. The ethical approvals for this project, including informed consent from the patients, were granted following the guidelines of the Hospital Clínic Ethics Committee (Institutional Review Board, registration number 2012/7498).

\section{Cell lines}

Thirteen DLBCL cell lines from both GCB and $A B C$ subtypes were used in this study. SUDHL-4, SUDHL-6, SUDHL-8, SUDHL-16, NUDHL-1 and U2932 cell lines were purchased from the Deutsche Sammlung von Mikroorganismen und Zellkulturen (DSMZ). SUDHL-2 and WSU-DLCL2 were obtained from the American Type Culture Collection (ATCC) cell bank (LGC Standards). OCI-LY8 and Toledo were kindly provided by Dr M. Raffeld (National Cancer Institute, Bethesda, MD, USA) and Dr MA Piris (Fundación Jiménez Díaz, Madrid, Spain). OCI-LY3 and OCI-LY10 cells were provided by Dr A. Staiger (Dr. Margarete Fischer-Bosch Institute of Clinical Pharmacology, Stuttgart, Germany). HBL-1 was provided by Dr E Valls (Transcriptional regulation of gene expression group, IDIBAPS, Barcelona, Spain). Cell lines were authenticated upon reception by short tandem repeat profiling, using an AmpFISTR identifier kit (Thermo Fisher Scientific), and based on available short tandem repeat profiles. All cell lines were cultured routinely at $37^{\circ} \mathrm{C}$ in a humidified atmosphere with 5\% carbon dioxide in RPMI 1640 or Iscove modified Dulbecco medium supplemented with $10 \%$ to $20 \%$ heat-inactivated fetal bovine serum, $2 \mathrm{mM}$ glutamine and $50 \mu \mathrm{g} / \mathrm{mL}$ penicillin-streptomycin (Thermo Fisher). Mycoplasm infection was routinely tested for by polymerase chain reaction. SUDHL-6 cells expressing green fluorescent protein (GFP) and Luciferase reporter genes, were generated as previously reported..$^{30}$

\section{Immunohistochemistry}

Fifty-two DLBCL samples were included in tissue microarrays using duplicated cores of $1 \mathrm{~mm}$ per tumor sample. CD31+ microvascular density was stained and quantified as previously described. ${ }^{31}$ Microvascular density values were grouped in quartiles and considered high or low when above or below the $50^{\text {th }}$ percentile. Xenograft tumor samples were stained for phosphohistone $\mathrm{H} 3$, cleaved caspase- 3 and MYC, as previously described. ${ }^{32}$ Phospho-Akt was detected using a monoclonal antiphospho-Akt-Ser473 antibody (Cell Signaling Technology). Preparations were evaluated on a DP70 or a BX51 microscope using Cell B Basic Imaging Software (Olympus).

\section{Western blot analysis}

Whole cell proteins were extracted from $10^{7}$ DLBCL cells as previously described. ${ }^{28}$ Proteins (30-50 $\left.\mu \mathrm{g} / \mathrm{lane}\right)$ were subjected to $10-12 \%$ sodium dodecylsulfate-polyacrylamide gel electrophoresis, and transferred onto polyvinylidene difluoride 
membranes (Immobilon-P; Millipore), or nitrocellulose membranes (Amersham Biosciences). Membranes were then incubated with primary and secondary antibodies (Online Supplementary Materials and Methods) and visualized on a mini-LAS4000 device (Fujifilm) by enhanced chemiluminescence (ECL, Amersham Life Science).

\section{Flow cytometry analysis}

For the detection of surface CXCR4, DLBCL cells $\left(2 \times 10^{5}\right)$ were stained with a phycoerythrin (PE)-labeled anti-CXCR4 or isotype control antibody (BD Biosciences). For the CXCR4 occupancy assay, cells were pretreated for $1 \mathrm{~h}$ with IOS-01.01, AMD3100 (Sigma-Aldrich) or an anti-human CXCR4 control antibody, followed by sCXCR4 staining as above. For quantification of apoptosis, cells were labeled with Pacific Blue-conjugated annexin V (Thermo Fisher). A total of 10,000 events were acquired and analyzed on an Attune acoustic focusing cytometer (Thermo Fisher). The mean fluorescence intensity ratio was calculated as the ratio between the median fluorescence intensity of the CXCR4-labeled sample and that of the isotype.

\section{Chemotaxis assay}

DLBCL cells $\left(5 \times 10^{6}\right.$ cells $\left./ \mathrm{mL}\right)$ were cultured for $1 \mathrm{~h}$ in culture medium not containing fetal bovine serum but supplemented with $0.5 \%$ bovine serum albumin (Sigma-Aldrich), in the presence or absence of $100 \mu \mathrm{M}$ AMD3100 or IOS-01.01RS, and analyzed for CXCL12-dependent chemotaxis, as previously described. ${ }^{28}$ Values are referred to cells cultured without CXCL12.

\section{Cell proliferation assay}

DLBCL primary cultures and cell lines $\left(5 \times 10^{4}\right.$ cells) were incubated for 24-48 h with IOS-01.01RS, AMD3100 and/or CPI203 (kindly provided by Constellation Pharmaceuticals) at the indicated doses. MTT (3-(4,5-dimethylthiazolyl-2)-2,5-diphenyltetrazolium bromide) reagent (Sigma-Aldrich) was added for 1-5 additional hours before spectrophotometric measurements. Each measurement was made in triplicate. Values are represented using untreated control cells as the reference. Combination indexes were calculated using Calcusyn software version 2.0 (Biosoft). The interaction between two drugs was considered synergistic when the combination index was less than 0.8 .

\section{In vivo assays}

Prior to efficacy testing, an acute toxicity assay was conducted in NSG mice exposed to IOS-01.01RS salt or AMD3100. Animals ( 2 adult males and 2 adult females per dose) received a single administration of vehicle, IOS01.01-RS (per os) or plerixafor (intraperitoneally) at doses ranging from 2 to $10 \mathrm{mg} / \mathrm{kg}$, and were monitored during the first $4 \mathrm{~h}$ after administration, and daily for 2 weeks, for viability/mortality and vital parameters. This toxicity assay defined a maximum non-lethal dose of 5 $\mathrm{mg} / \mathrm{kg}$ for AMD3100, while the maximum lethal dose of IOS01.01RS remained unreached.
For the systemic DLBCL model, 8-week old NSG female mice $(\mathrm{n}=12)$ were inoculated intravenously with $10^{7}$ SUDHL6-GFPLuc cells, randomly assigned into three equivalent cohorts, and treated daily with $5 \mathrm{mg} / \mathrm{kg}$ AMD3100 (intraperitoneally), 10 $\mathrm{mg} / \mathrm{kg}$ IOS-01.01RS salt (per os) or vehicle. After 27 days, animals were sacrificed and peripheral blood was collected by submandibular puncture. Erythrocytes were lysed using ACK buffer (Quality Biological) and the percentage of SUDHL- 6 cells was evaluated by detection of a GFP signal on an Attune cytometer. For the subcutaneous DLBCL model, SUDHL6-GFP-Luc cells were inoculated subcutaneously in 8-week old NSG female mice as previously. ${ }^{28,32}$ Animals were randomly assigned into four cohorts of four mice each and were given a twice daily dose of $1.5 \mathrm{mg} / \mathrm{kg}$ CPI203 (intraperitoneally), a daily dose of $2 \mathrm{mg} / \mathrm{kg}$ IOS-01.01RS (per os), both agents, or an equal volume of vehicle. Tumor engraftment was determined weekly following mice injection with $75 \mathrm{mg} / \mathrm{kg}$ D-luciferine (AnaSpec) and bioluminescence imaging (BLI) using an Aequoria Luxiflux device equipped with an ORCA-ER camera (Hamamatsu). The bioluminescence imaging signal was quantified using Image J software. Tumor volume was measured by external calipers twice a week, up to 28 days. Animals were then sacrificed and tumors were harvested and weighed. Animals were handled following protocols approved by the Animal Ethics Committee of the University of Barcelona (protocol \#154/16).

\section{Statistical analysis}

A Wilcoxon rank-sum test, Fisher exact test, Spearman rank correlation or $t$-test was used to examine the statistical significance of associations between clinico-pathological data and CXCL12-CXCR4 positivity or cytokine expression level, as appropriate. Survival curves were estimated using the KaplanMeier method. A log-rank test was used to compare survival curves between groups. For in vitro and in vivo functional assays, unpaired and paired $t$-tests were employed to obtain the statistical significance. The Benajmini-Hochberg method was used to adjust $P$-values for multiple testing. Results were considered statistically significant when the $P$ value was less than 0.05

\section{Results}

\section{CXCL12 expression correlates with microvascular density and confers an unfavorable prognosis to patients with diffuse large B-cell lymphoma}

Gene expression profiling studies have highlighted the prognostic value of the microenvironment and components of several immune regulatory pathways in DLBCL. ${ }^{4}$ To characterize which cytokines may have a significant impact on the pathogenesis of DLBCL, we performed a fluorescence-based cytokine antibody array using frozen tissue from 12 DLBCL cases, out of an initial cohort of 52 patients (Online Supplementary Table S1), and correlated expression data with clinico-biological characteristics of

Table 1. Top cytokines associated with high microvessel density in diffuse large B-cell lymphoma biopsies.

\begin{tabular}{|c|c|c|c|c|c|c|}
\hline Cytokine & $\begin{array}{c}\text { Mean } \\
\text { (MVD=low) }\end{array}$ & $\begin{array}{c}\text { Mean } \\
\text { (MVD=high) }\end{array}$ & Difference & t statistic & $\mathbf{P}$ & Adjusted P \\
\hline SDFl $\alpha / C X C L 12$ & 5.207 & 5.637 & 0.430 & -3.758 & 0.000 & 0.032 \\
\hline IGFBP2 & 4.467 & 4.946 & 0.479 & -3.744 & 0.000 & 0.032 \\
\hline IGFBP1 & 5.085 & 5.601 & 0.516 & -3.666 & 0.001 & 0.032 \\
\hline
\end{tabular}

MVD: microvessel density. 
the patients. Among the 175 cytokines included in this array, we found that only CXCL12, IGFBP2 and IGFBP3 correlated significantly with high microvascular density $(P<0.05)$ (Table 1$)$, a parameter associated with an unfavorable prognosis in DLBCL. ${ }^{31,33}$ Additionally, high expression of CXCL12 correlated significantly with bone marrow involvement at diagnosis $(P=0.02$, data not shown), thus supporting a role for the CXCL12-CXCR4 axis in the progression of DLBCL.

\section{IQS-01.01RS is a novel CXCR4 inhibitor with improved pharmacological properties}

To evaluate the therapeutic potential of targeting CXCR4 in DLBCL, we took advantage of a novel family of CXCR4 inhibitors with improved pharmacodynamic properties and lower cardiotoxicity than the standard CXCR4 inhibitor AMD3100..$^{34,35}$ Similarly to AMD3100, IOS-01.01 is a symmetric molecule with a central p- phenylene group harboring two chiral carbons. The compound thus comports three stereoisomers, namely IOS01.01RR, IOS-01.01SS and IOS-01.01RS (Figure 1A). Nitrogen atoms on each site are intercalated in carbon chains at a similar distance as those of the cyclam in AMD3100 providing IOS-01.01 with positively charged nitrogen atoms at physiological $\mathrm{pH}$ which, as with AMD3100, will interact with the lateral chains of acidic amino acids of CXCR4, but with greater flexibility. We first assessed the inhibitory activity of IOS-01.01 (racemic mixture), and its three individually purified stereoisomers, using a CXCR4 antagonist screening study. AMD3100 was used as a reference drug. As shown in Figure $1 \mathrm{~B}$, the three stereoisomers had superior CXCR4 antagonist activity to that of AMD3100, with IQS01.01RS (see structure in Figure 1C) being the most potent agent. This compound led to a $181 \%$ inhibition of receptor activity, thus resembling an inverse receptor ago-
A<smiles>CC1CCCCN1CCCNCc1ccc(CNCCCN2CCCC[C@H]2C)cc1</smiles>

C

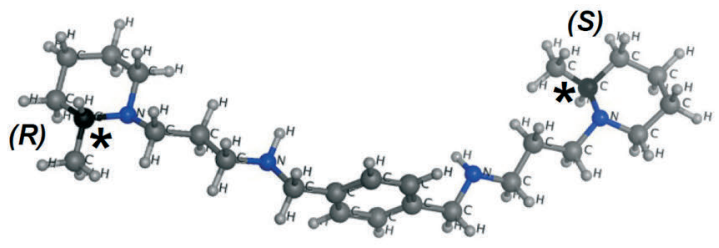

D

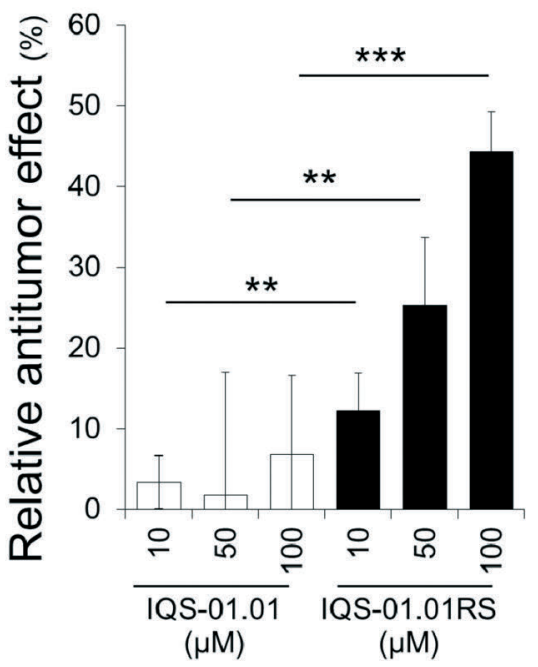

B

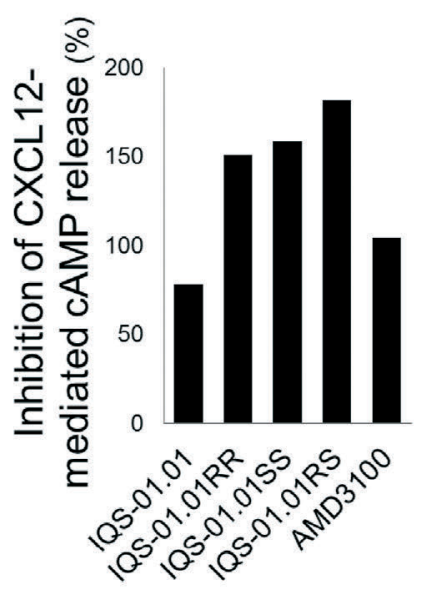

$\mathbf{E}$

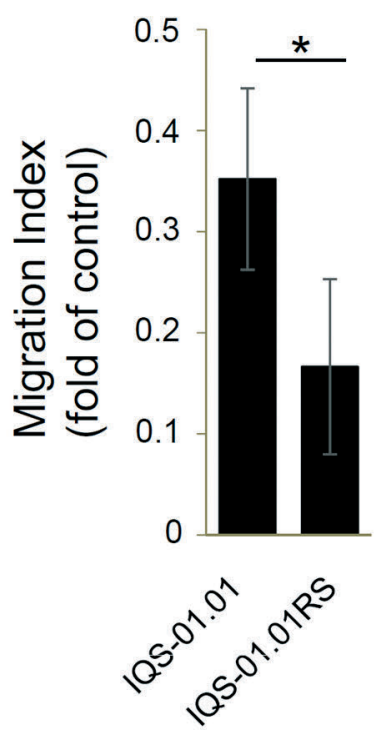

Figure 1. Design of a new potent inhibitor of CXCR4. (A) Skeletal structure of IQS-01.01. *chiral carbons. (B) Inhibition of CXCL12-mediated intracellular cAMP release was determined in the presence of a racemic mixture of IQS-01.01 and its three individually purified stereoisomers, using AMD3100 blocking activity as a reference control. (C) Ball-and-stick representation of IQS-01.01RS. * chiral carbons. (D) MTT assay showing superior antitumor effect of IQS-01.01RS compared to that of the IQS-01.01 racemic mixture after $48 \mathrm{~h}$. The graph shows mean values obtained from three GCB-DLBCL cell lines (SUDHL6, SUDHL-16, and WSU-DLCL2) and three $A B C-D L B C L$ cell lines (OCI-LY3, OCI-LY10 and SUDHL-2). (E) Inhibition of CXCL12-induced migration upon DLBCL cell treatment with a $100 \mathrm{uM}$ dose of IOS01.01 racemic mixture or IQS-01.01RS. Mean values for the SUDHL-6 and OCI-LY3 cell lines are shown. ${ }^{\star} P<0.05, * * P<0.01, * * * P<0.001$. 
A

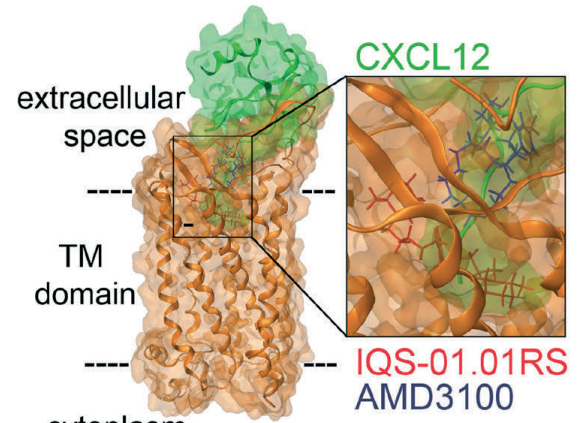

D

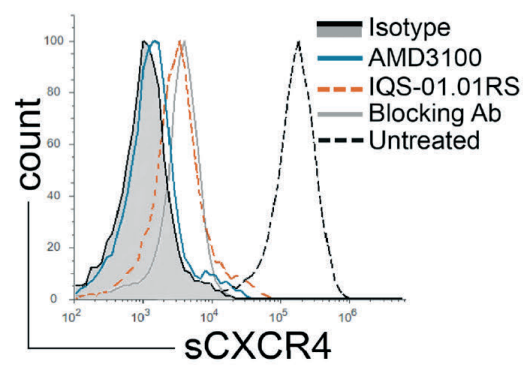

E

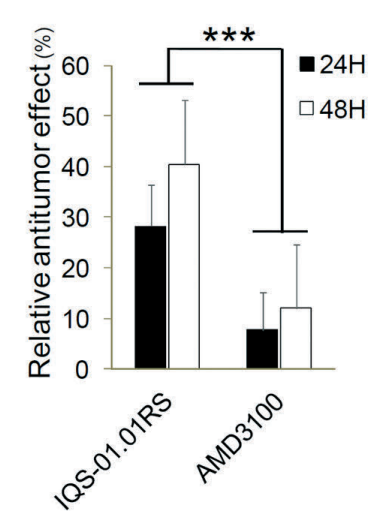

$\mathbf{F}$

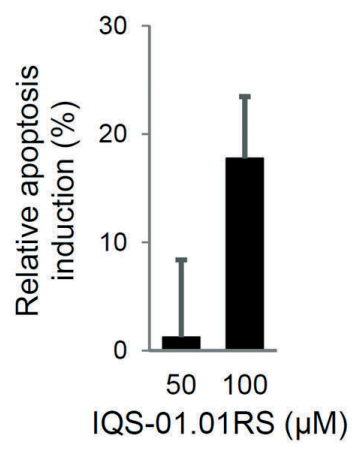

D

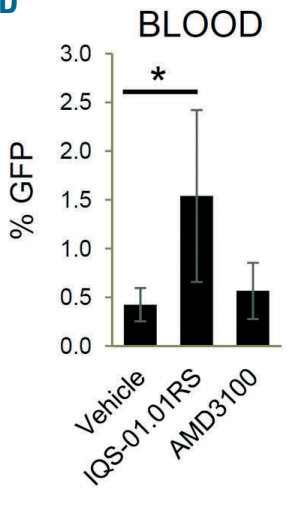

G

SUDHL-6

QQS-01.01RS AMD3100

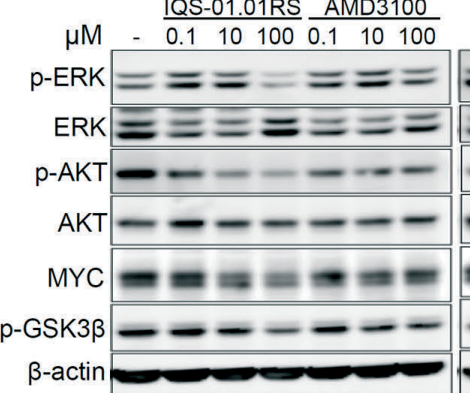

U2932 $\frac{\text { IQS-01.01RS }}{0.110100} \frac{\text { AMD3100 }}{0.110100}$

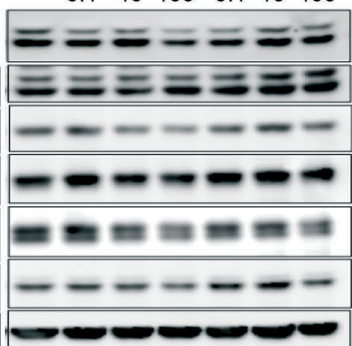

H

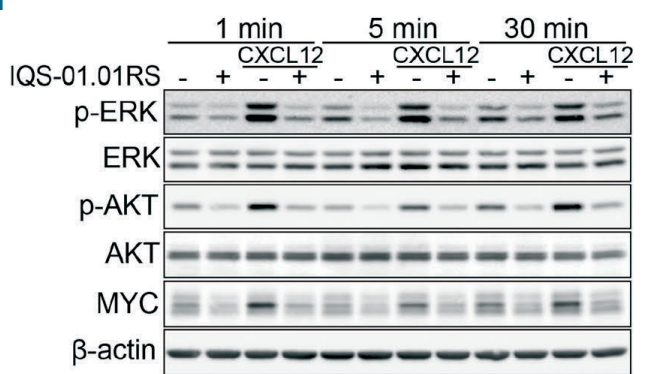

Figure 2. IQS-01.01RS has better pharmacological properties than those of AMD3100. (A) Predicted docking of IQS-01.01RS (red) and AMD3100 (blue) on their target, CXCR4 (orange). CXCL12 is represented in green. (B) A CXCR4 occupancy assay shows the competition between IQS-01.01RS or AMD3100 (100 uM) with a phycoerythrin-labeled anti-CXCR4 antibody for binding to the receptor. A blocking antibody (30 $\mathrm{g} / \mathrm{mL}$, R\&D Systems) was used as a control. (C) Inhibition of CXCL12induced migration upon DLBCL cell treatment with increasing doses of IQS-01.01RS or AMD3100. The graphs show mean values from three GCB-DLBCL cell lines (SUDHL8, Toledo and SUDHL-6) and two ABC-DLBCL cell lines (U2932 and OCI-LY3). Data are representative of at least three independent experiments. (D) Mean percentage of tumor B cells detected in blood samples of NSG mice injected intravenously with SUDHL-6-GFP-LUC cells and treated for 27 days with IQS-01.01RS, AMD3100, or vehicle ( $n=4$ animals/group). (E) Time-dependent antitumor effect of IOS-01.01RS (100 uM) and AMD3100 (100 $u$ M) in a panel of 13 DLBCL cell lines; the effect was determined by a MTT assay. Representative results from three experiments are shown. (F) Relative induction of apoptosis in CD $19^{+}$tumor B cells upon treatment of primary DLBCL biopsies $(n=5)$ with the indicated doses of IQS-01.01RS for $48 \mathrm{~h}$. The mean viability of untreated primary cells was $79 \pm 8 \%$. (G) Western blot analysis of CXCR4 downstream signaling in SUDHL6 and U2932 cells upon $2 \mathrm{~h}$ starvation, followed by exposure to recombinant CXCL12 for 1 min, with or without pretreatment with the indicated doses of IQS-01.01RS or AMD3100. $\beta$-actin was used as a loading control. (H) CXCR4 downstream signaling and MYC modulation in SUDHL-6 cells at different time points, after $2 \mathrm{~h}$ starvation followed by receptor triggering in the presence or absence of $100 \mu \mathrm{M}$ IQS-01.01RS. $\beta$-actin was used as a loading control. Ab: antibody: TM: transmembrane. $* P<0.05, * * * P<0.001$. 
Table 2. Sensitivity of diffuse large B-cell lymphoma cell lines to CXCR4 and BET bromodomain inhibition.

\begin{tabular}{|c|c|c|c|c|c|}
\hline Sulbtype & Cell line & $\begin{array}{l}\text { CXCR4 expression } \\
\text { (MFI-R) }\end{array}$ & $\begin{array}{l}\text { IQS-01.01RS } \\
\text { cytotoxic effect } \\
\text { (100 pM) }\end{array}$ & $\begin{array}{c}\text { CP1203 } \\
\text { cytotoxic effect } \\
(0.5 \mathrm{MM})\end{array}$ & $\begin{array}{l}\text { Combination } \\
\text { index }\end{array}$ \\
\hline $\mathrm{GCB}$ & $\begin{array}{c}\text { OCI-LY8 } \\
\text { SUDHL-4 } \\
\text { SUDHL-6 } \\
\text { SUDHL-16 } \\
\text { WSU-DLCL2 } \\
\text { Toledo } \\
\text { SUDHL-8 } \\
\text { NUDHL-1 }\end{array}$ & $\begin{array}{c}36 \\
25 \\
41 \\
1 \\
3 \\
52 \\
91 \\
1\end{array}$ & $\begin{array}{c}32 \% \\
37 \% \\
42 \% \\
36 \% \\
35 \% \\
9 \% \\
38 \% \\
36 \%\end{array}$ & $\begin{array}{l}38 \% \\
59 \% \\
47 \% \\
86 \% \\
55 \% \\
43 \% \\
14 \% \\
60 \%\end{array}$ & $\begin{array}{l}1.1 \\
0.6 \\
0.4 \\
0.5 \\
0.4 \\
0.5 \\
1.1 \\
0.8\end{array}$ \\
\hline $\mathrm{ABC}$ & $\begin{array}{c}\text { OCI-LY3 } \\
\text { OCI-LY10 } \\
\text { SUDHL-2 } \\
\text { U2932 } \\
\text { HBL-1 }\end{array}$ & $\begin{array}{c}4 \\
1 \\
1 \\
46 \\
1\end{array}$ & $\begin{array}{l}48 \% \\
40 \% \\
53 \% \\
43 \% \\
63 \%\end{array}$ & $\begin{array}{l}75 \% \\
40 \% \\
95 \% \\
31 \% \\
39 \%\end{array}$ & $\begin{array}{l}0.7 \\
0.8 \\
0.8 \\
0.9 \\
0.6\end{array}$ \\
\hline
\end{tabular}

ABC: activated B-cell; GCB: germinal center B-cell; MFI-R: median fluorescence intensity ratio.

nist. We then analyzed the antitumor activity of this highly active stereoisomer, together with IOS-01.01 (racemic mixture) in six representative DLBCL cell lines of both GCB and ABC subtypes. As shown in Figure 1D, IOS-01.01RS was significantly more active than the racemic mixture at all the doses tested, with the greatest effect being observed at the $100 \mu \mathrm{M}$ dose (mean cytotoxicity: 44\%; range, 42-49\%). This effect was mainly attributed to proliferation blockade, as IOS-01.01RS-treated cultures showed low $(<5 \%$ ) levels of apoptosis (data not shown). In agreement with this, migration experiments using a CXCL12 gradient further demonstrated that IOS01.01RS was a more potent inhibitor of cell chemotaxis than was the racemic mixture, with a 2-fold improvement of cell migration blockade (Figure 1E). Based on these results, we proceeded to a deeper study of the stereoisomer of choice, IOS-01-01RS.

A predictive docking model showed that this stereoisomer localized into the extracellular CXCL12 binding domain of CXCR4, close to the position occupied by AMD3100, and simultaneously interacted with an inner, transmembrane domain of the receptor. This unique feature potentially provides IOS-01.01RS with the ability to inhibit CXCR4 in the presence of the ligand, and to interfere with the catalytic activity of the receptor (Figure 2A). Supporting this model, using the representative cell line SUDHL-6 we observed that IOS-01.01RS and AMD3100 had similar CXCR4 occupancy activity (Figure 2B), and had similar anti-migratory properties against a CXCL12 gradient in vitro, both in ABC- and GCB-DLBCL cell lines (Figure 2C). However, IOS-01.01RS triggered superior mobilization of DLBCL cells into the circulating blood of the animals (Figure 2D) and was able to block the proliferation of a panel of 13 GCB/ABC-DLBCL cell lines in a time-dependent manner, contrasting with the modest antitumor activity of $\mathrm{AMD} 3100$ (40\% versus $12 \%$ mean cell growth inhibition at $48 \mathrm{~h}$ ) (Figure $2 \mathrm{E}$ and Table 2). In addition, in a set of five DLBCL primary cultures, a dosedependent induction of apoptosis was observed upon exposure to IOS-01.01RS (Figure 2F), contrasting with the reported inability of AMD3100 to induce cell death. ${ }^{36}$ Accordingly, the analysis of CXCR4 downstream signaling showed that the capacity of IOS-01.01RS to inhibit basal and CXCL12-induced phosphorylation of ERK1/2,
AKT and the AKT downstream kinase GSK3- $\beta$, was superior to that of AMD3100 (Figure 2G). Of special interest, IQS-01.01RS, rather than AMD3100, allowed strong downregulation of the MYC proto-oncogene in ABC- and GCB-DLBCL cells (Figure 2H). This downstream target of CXCR4 with a well-established role in the progression of DLBCL, ${ }^{37}$ has been reported to depend on AKT phosphorylation level for its stabilization, mediated by a GSK3- $\beta$-dependent phosphorylation of its Thr58 residue and consequent proteosomal degradation. ${ }^{38,39}$ Supporting the hypothesis that the downregulation of MYC may be consequent to inhibition of the CXCR4-AKT axis in IOS-01.01RS-treated cells, the expression of both p-AKT and MYC was almost completely abrogated within a few minutes after the addition of IOS-01.01RS (Figure 2H) and this phenomenon could be maintained for at least $3 \mathrm{~h}$ (data not shown). Collectively, these results indicate that the new CXCR4 inhibitor IOS-01.01RS has a unique structure that enables its binding to two distinct domains of the receptor, conferring improved mobilizing properties in vivo, as well as superior antitumor activity in vitro.

\section{IQS-01.01RS cooperates with BET bromodomain inhibition in vitro and in vivo}

Based on our observation that IOS-01.01RS had the capacity to downregulate MYC, we further investigated in our 13 DLBCL cell lines whether the compound could cooperate with the BET bromodomain antagonist CPI $203^{32}$ to block cell proliferation. The combination of each agent induced up to $78 \%$ cytoxicity at the optimal doses of $100 \mu \mathrm{M}$ IQS-01.01RS and $500 \mathrm{nM}$ CPI203, with a mean $\mathrm{CI}$ of 0.67 , indicative of a synergistic drug interaction in both ABC- and GCB-DLBCL cells (Figure 3A and Table 2). As expected, a dramatic reduction in MYC protein levels was observed after exposure to the combination (Figure 3B). We confirmed by quantitative polymerase chain reaction analysis and cycloheximide protein stability assays that this phenomenon was consequent to a simultaneous CPI203-evoked transcriptional repression of MYC gene and an IOS-01.01RS-mediated destabilization of MYC protein (Figure 3C,D). Confirming that this effect was not due to a BRD4 inhibitor-mediated diminished production of CXCR4, as previously described in T 
lymphocytes following JQ1 treatment, ${ }^{40}$ Figure 3B shows that CPI203 addition did not alter CXCR4 protein levels either in the presence or absence of IOS-01.01RS. Flow cytometry analysis further confirmed that surface levels of CXCR4 were unmodified by BET bromodomain inhibition (data not shown). To further ascertain the role of CXCR4 expression in this drug interaction, we established genetic depletion of CXCR4 in SUDHL-6 cells employing a CRISPR/Cas9 gene editing tool to generate CXCR4 knocked out (KO) cell lines, prior to treatment with CPI203, IOS-01.01RS and the combination of both agents. In parallel, and as a control of non-lymphomatous B malignancy, we used the recently described NALM6CXCR4-KO cell line (kindly provided by Dr Jan Burger, Department of Leukemia, The University of Texas MD Anderson Cancer Center, Houston, TX, USA). As shown in Online Supplementary Figure S1, CXCR4-KO cell lines completely lack CXCR4 molecules on their surface. The main difference between these two cell lines was the response to CPI203 as a single agent, which was substantially weaker in NALM6-CXCR4-KO cells. This may illustrate the recent finding that, in acute myeloid leukemia cells, CXCR4 signaling is involved in the regulation of $M Y C$ transcription mediated by the downregulation of the miRNA let-7a ${ }^{41}$ while in DLBCL cells no variation in MYC mRNA levels are observed following CXCR4 ligation (Online Supplementary Figure S2). Another explanation may come from the lower membrane expression (about one $\log _{10}$ ) of the receptor observed in parental acute myeloid leukemia cells when compared to parental DLBCL cells, suggesting that NALM6 cells are less dependent on CXCR4 and less susceptible to CXCR4dependent cellular stress than are SUDHL-6 cells. Most importantly, in the acute myeloid leukemia cell line the
A

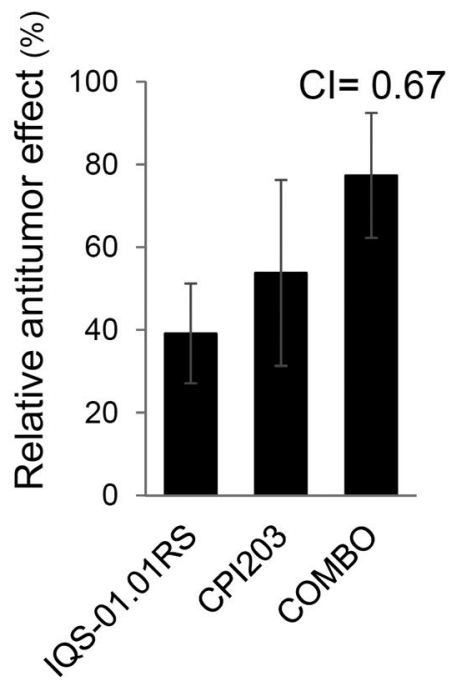

C

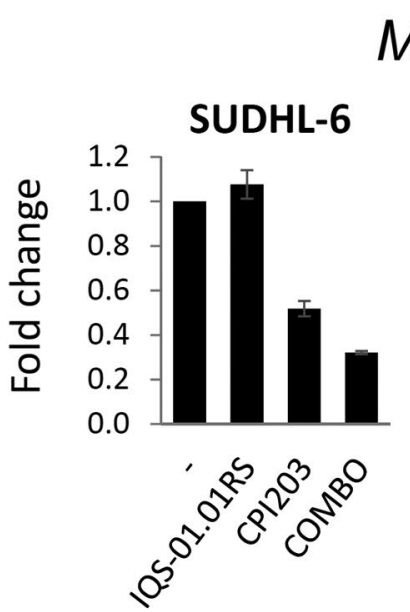

MYC

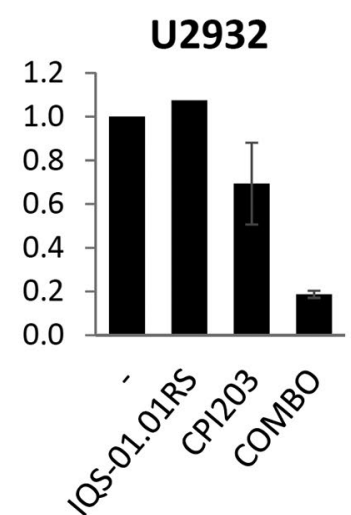

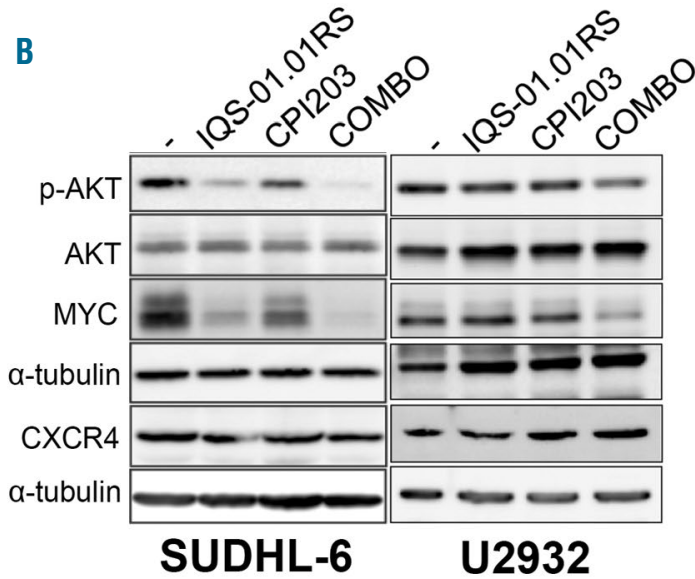

D

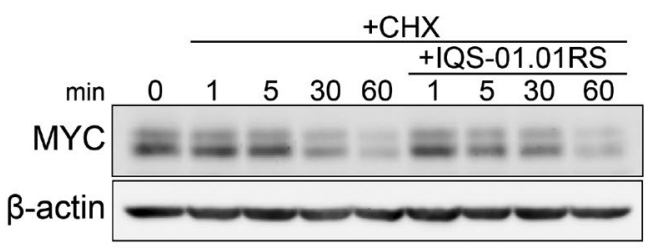

Figure 3. IQS-01.01RS synergizes with the BET bromodomain inhibitor CPI203 in vitro. (A) The relative antitumor effect of IQS-01.01RS (100 $\mu \mathrm{M})$, CPI203 (0.5 $\mu \mathrm{M})$ and the combination of both was determined by a MTT assay, after $48 \mathrm{~h}$. The data shown are the mean results of the $13 \mathrm{DLBCL}$ cell lines. (B) Cooperation between IQS-01.01RS and CPI203 in the inhibition of CXCR4 downstream signaling, as assessed by western blot analysis of p-AKT and MYC. SUDHL-6 and U2932 cells were starved for $2 \mathrm{~h}$, and treated for $1 \mathrm{~h}$ with $100 \mu \mathrm{M}$ IQS-01.01RS and/or CPI203 (0.5 $\mu \mathrm{M})$ prior to a $1 \mathrm{~min}$ stimulation with $200 \mathrm{ng} / \mathrm{mL}$ recombinant CXCL12. $\alpha$-tubulin was used as a loading control. (C) Relative MYC transcript levels in SUDHL-6 and U2932 cells upon $6 \mathrm{~h}$ treatment with $100 \mu \mathrm{M}$ IQS-01.01RS, $0.5 \mu \mathrm{M}$ CPI203 and the combination of both. Control untreated cells were used as a reference. (D) Time-dependent determination of MYC protein levels in SUDHL-6 cells treated with the translational blocker cycloheximide, as previously described, ${ }^{62}$ in the presence or absence of $100 \mu \mathrm{M}$ IQS01.01-RS. $\beta$-actin was used as a loading control. $\mathrm{Cl}$ : combination index; CHX: cycloheximide; COMBO: combination treatment with IQS-01.01RS and CPI203. 
loss of CXCR4 was associated with $30 \%$ and $24 \%$ decreases in the cytotoxic effect of CPI203 and the CPI203/IQS-01.01RS combination, respectively, confirming that CXCR4 expression was required for the activity of the bromodomain inhibitor alone, as well as for its synergistic activity with IOS-01.01RS. In the case of SUDHL6, although the combination was still active in CXCR4-depleted cells, the loss of the receptor induced a $13 \%$ reduction in the synergistic activity of the two agents when compared with the parental CXCR4 $4^{+}$cells.
Thus, these results strongly support a crucial role for CXCR4 in the cooperation between CPI203 and IOS01.01RS in malignant B cells.

To assess the efficacy of the drug combination in vivo, NSG mice were subcutaneously injected with SUDHL6GFP-Luc cells, and given IQS-01.01RS, CPI203, the combination of both agents, or the equivalent volume of vehicle, for 13 days. Tumor burden was evaluated weekly by bioluminescence signal recording and twice a week by external calipers. At the final time point, tumors were
A

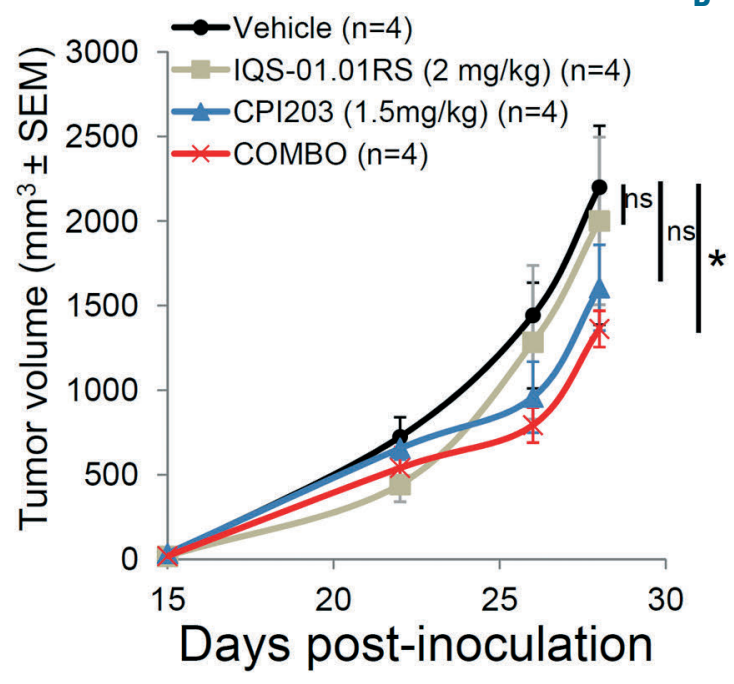

week 3 week 4

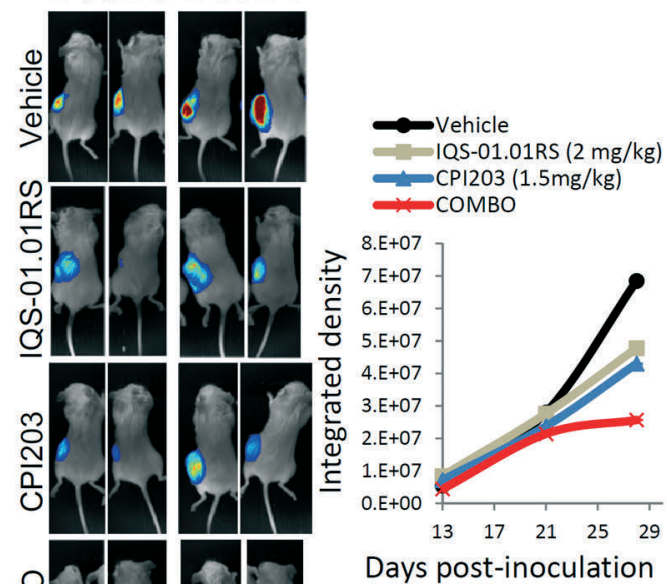

C

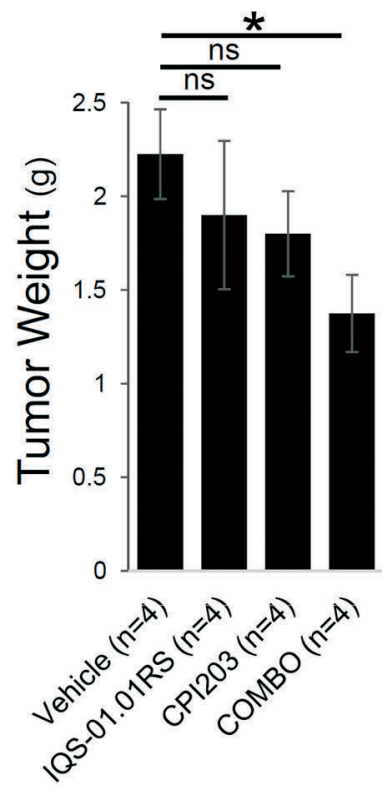

D

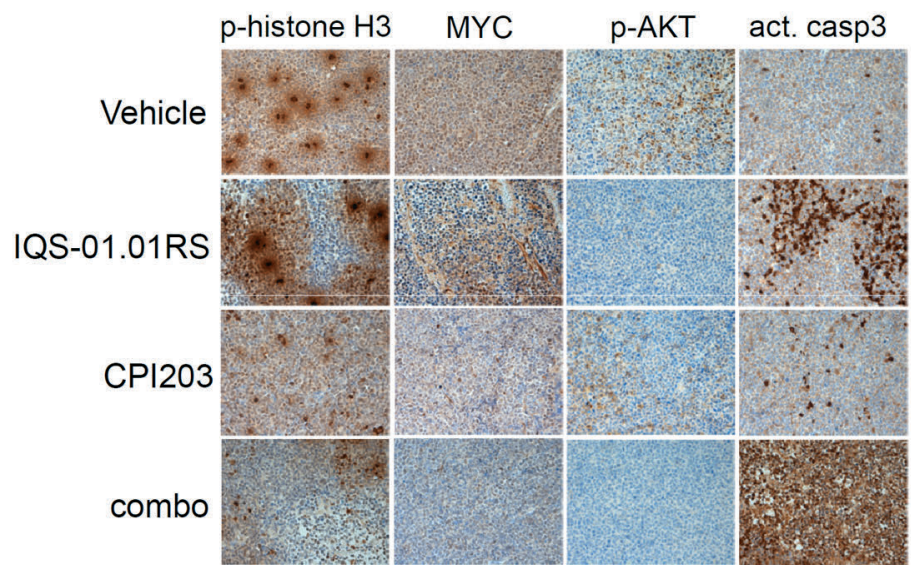

Figure 4. IQS-01.01RS and CPI203 cooperate to reduce tumor growth in a subcutaneous mouse model of diffuse large B-cell lymphoma. NSG mice were subcutaneously injected with SUDHL6-GFP'Luc ${ }^{+}$cells and tumor-bearing mice were randomly assigned to one of the following treatment arms (4 mice per group): IQS01.01RS $2 \mathrm{mg} / \mathrm{kg}$ daily (per os), CPI203 $1.5 \mathrm{mg} / \mathrm{kg}$ BID (intraperitoneally), both agents or equal volume of vehicle, for 2 weeks. (A) Tumor volume was evaluated twice a week using external calipers. (B) Tumor burden was evaluated at week 3 and week 4 by analysis of the bioluminescence signal. Left panel: color maps of two representative animals per group. Right panel: quantification of luciferase activity using Image J software. (C) Mean tumor weight in each treatment group at the final time point. (D) Immunohistochemical labeling of $\mathrm{p}$-histone $\mathrm{H} 3$, activated caspase-3, MYC and p-AKT in consecutive tissue sections from four representative tumor specimens (magnification $\times 200$ ). act casp3: activated caspase 3; COMBO/combo: combination treatment with IQS-01.01RS and CPI203; SEM: standard error of mean; ns=not significant; * $P<0.05$. 
extracted and weighed. Figure 4A shows that CPI203 and IOS-01.01RS as single agents induced $27 \%$ and $4 \%$ reductions in tumor growth, respectively, while the combination of both drugs induced a $38 \%$ decrease in tumor burden. Accordingly, reduced luciferase activity as well as a significant $(38 \%)$ decrease in tumor weight was detected in mice given combination treatment compared to the values in the group administered the vehicle (Figure $4 \mathrm{~B}, \mathrm{C})$. Histological analysis of the corresponding tumors confirmed a greater reduction of the mitotic index together with an accumulation of apoptotic cells by the combination therapy, as assessed by phospho-histone $\mathrm{H} 3$ and activated-caspase-3 staining (Figure 4D). In agreement with the in vitro results, greater reductions in MYC and phospho-Akt were observed in animals treated with the combination of drugs (Figure 5D). Collectively, these results suggest that the combination of IOS-01.01RS with the BET inhibitor CPI203 enhances the antitumor properties of each single agent through the blockade of CXCR4 signaling, followed by the abrogation of MYC expression and the induction of apoptosis.

\section{Discussion}

The chemokine receptor CXCR4 has a prominent role in homing and retention of tumor cells in their microenvironment, and in the promotion of drug resistance..$^{42}$ It has been previously reported that CXCR4/CXCL12 expression by tumor cells confers an adverse prognosis to DLBCL patients. ${ }^{12,43}$ In agreement with this, we observed that CXCL12 expression in tumor biopsies correlated with bone marrow involvement at diagnosis, as well as highly vascularized tumors. These observations support the hypothesis that the CXCL12-CXCR4 axis may play a significant role in neo-angiogenesis in the DLBCL microenvironment. Accordingly, various reports have highlighted a correlation between CXCR4 expression and high serum levels of VEGF in different cancer subtypes. ${ }^{19,21,44}$ CXCL12 is also one of the genes included in the DLBCL pro-angiogenic and unfavorable "stromal-2 signature" ${ }^{4}$ while activation of the CXCL12-CXCR4 axis is related to tumor angiogenesis and recruitment of endothelial progenitor cells to tumors in myelodysplastic syndrome, glioma and pancreatic cancer. ${ }^{20,45,46}$

The CXCL12-CXCR4 axis may, therefore, represent a new therapeutic target for DLBCL, as inhibition of CXCR4 and tumor cell mobilization is bound to increase the accessibility of these lymphomas to anticancer therapies. Validating this strategy, the Food and Drug Administration-approved drug AMD3100 has been shown to potentiate the tumor growth inhibition afforded by standard chemotherapeutics and/or irradiation in preclinical models of glioblastoma, ${ }^{26,47}$ to inhibit the engraftment of B-cell acute lymphoblastic leukemia, ${ }^{48}$ to mobilize and sensitize acute promyelocytic leukemia cells to cytosine arabinoside, ${ }^{49}$ and to enhance the response to rituximab and to the anti-CD52 monoclonal antibody alemtuzumab in a mouse model of disseminated lymphoma.$^{50}$ In the clinical setting, encouraging preliminary data have been obtained from a phase II study using AMD3100 and standard chemotherapy in patients with acute myeloid leukemia. ${ }^{51}$ In DLBCL, pharmacological inhibition of CXCR4 by AMD3100 impairs the propagation of tumor $\mathrm{B}$ cells in a systemic mouse model of the disease. ${ }^{12}$ Nonetheless, AMD3100 presents serious limitations that could preclude its use as an anticancer drug, such as some degree of cardiotoxicity and adverse pharmacodynamic properties including a positive charge at physiological $\mathrm{pH}$ and a limited half-life, impairing its oral bioavailability. ${ }^{52}$ Thus, the lack of a CXCR4 inhibitor suitable for administration in a standard regimen supports the development of less toxic and more stable CXCR4targeting agents..$^{53}$

Here, we describe the biological activity of IOS01.01RS, a new potential CXCR4 inhibitor with a higher lethal dose in vivo and better pharmacodynamic properties than AMD3100.. We report that by interacting with a different CXCR4 domain than that with which AMD3100 interacts, this IOS-01.01RS acts as a putative allosteric CXCR4 inhibitor, impeding the activation of the receptor upon CXCL12 ligation and allowing a more sustained inhibition of the pathway. In vivo, this effect, together with better pharmacokinetic properties, was linked to an improved capacity of the compound to mobilize DLBCL cells. Furthermore, we show that IOS01.01RS-mediated blockade of CXCR4 signaling led to the post-transcriptional downregulation of MYC. Overexpression of this oncogene is associated with shorter survival in DLBCL patients, ${ }^{54}$ and is known to be stabilized by CXCR4 in cancer cells..$^{55}$ MYC was considered to be an undruggable factor until (+)-JQ1, a highly potent, selective and cell-permeable inhibitor of BRD4, a member of the BET family of chromatin adaptors, was first shown to have antitumor activity in multiple myeloma xenograft models. ${ }^{56}$ Further studies with (+)-JO1 and CPI203, a molecule characterized by a superior bioavailability after oral or intraperitoneal administration, ${ }^{32,57}$ have validated $\mathrm{BET}$ bromodomain targeting and subsequent blockade of MYC, NF- $\mathrm{KB}$ or BCL-2 protein family signaling as a promising therapeutic strategy in different subtypes of aggressive B-cell lymphoma, including DLBCL. ${ }^{58-60}$ BRD4 is a global regulator of gene transcription which selectively recognizes and binds to acetylated lysine residues in histones to activate transcription and mitosis. In DLBCL it preferentially localizes in superenhancers associated with key transcription factors implicated in lymphomagenesis, such as MYC, which are specifically sensitive to BRD4 inhibition thus explaining the selective anti-tumor effect of BRD4 inhibitors. ${ }^{61} \mathrm{It}$ is worth pointing out that BET bromodomain inhibition, as a consequence, downregulates other tumor-related genes apart from MYC. ${ }^{61}$ Nevertheless, in this work, we focused on the capacity of CPI203 to inhibit MYC, confirming that ABC- and GCB-DLBCL cells are highly sensitive to CPI203 monotherapy, and that the transcriptional downregulation of $M Y C$ achieved by this compound allows almost complete abrogation of the protein when the compound is combined with IOS-01.01RS. This dual approach underlies the synergistic interaction of the two compounds and consequent sensitization to apoptosis in vitro and in vivo.

In conclusion, the present work shows that, besides CXCR4, the level of CXCL12 may also have an impact on the progression of DLBCL, and describes a new potent orally available CXCR4 inhibitor with antitumor properties. In addition, this study offers the first rational basis for the potential clinical evaluation of a dual approach combining BET bromodain inhibition and CXCR4 blockade in DLBCL. 


\section{References}

1. Teras LR, DeSantis CE, Cerhan JR, Morton LM, Jemal A, Flowers CR. 2016 US lymphoid malignancy statistics by World Health Organization subtypes. CA Cancer J Clin. 2016 Sep 12. [Epub ahead of print]

2. Miyazaki K. Treatment of diffuse large Bcell lymphoma. J Clin Exp Hematop. 2016;56(2):79-88.

3. Alizadeh AA, Eisen MB, Davis RE, et al. Distinct types of diffuse large B-cell lymphoma identified by gene expression profiling. Nature. 2000;403(6769):503-511

4. Lenz G, Wright G, Dave SS, et al. Stromal gene signatures in large-B-cell lymphomas. N Engl J Med. 2008;359(22):2313-2323.

5. Shain KH, Dalton WS, Tao J. The tumor microenvironment shapes hallmarks of mature B-cell malignancies. Oncogene. 2015;34(36):4673-4682.

6. Allen CDC, Ansel KM, Low C, et al. Germinal center dark and light zone organization is mediated by CXCR4 and CXCR5. Nat Immunol. 2004;5(9):943-952.

7. Blades MC, Manzo A, Ingegnoli F, et al. Stromal cell-derived factor 1 (CXCL12) induces human cell migration into human lymph nodes transplanted into SCID mice. J Immunol. 2002;168(9):4308-4317.

8. Foudi A, Jarrier P, Zhang Y, et al. Reduced retention of radioprotective hematopoietic cells within the bone marrow microenvironment in CXCR4-/- chimeric mice. Blood. 2006;107(6):2243-2251.

9. Peled A, Petit I, Kollet O, et al. Dependence of human stem cell engraftment and repopulation of NOD/SCID mice on CXCR4. Science. 1999:283(5403):845-848.

10. Peled A, Kollet $\mathrm{O}$, Ponomaryov T, et al. The chemokine SDF-1 activates the integrins LFA-1, VLA-4, and VLA-5 on immature human $\mathrm{CD} 34(+)$ cells: role in transendothelial/stromal migration and engraftment of NOD/SCID mice. Blood. 2000;95(11):32893296.

11. Scala S. Molecular pathways: targeting the CXCR4-CXCL12 axis--untapped potential in the tumor microenvironment. Clin Cancer Res. 2015;21(19):4278-4285.

12. Moreno MJ, Bosch R, eguez-Gonzalez R, et al. CXCR4 expression enhances diffuse large B cell lymphoma dissemination and decreases patient survival. J Pathol. 2015;235(3):445-455

13. Zlotnik A, Burkhardt AM, Homey B. Homeostatic chemokine receptors and organ-specific metastasis. Nat Rev Immunol. 2011;11(9):597-606.

14. Bleul CC, Fuhlbrigge RC, Casasnovas JM, Aiuti A, Springer TA. A highly efficacious lymphocyte chemoattractant, stromal cellderived factor 1 (SDF-1). J Exp Med. 1996;184(3):1101

15. Nagasawa T. The chemokine CXCL12 and regulation of HSC and B lymphocyte development in the bone marrow niche. Adv Exp Med Biol. 2007;602(69-75

16. Burger JA, Kipps T]. Chemokine receptors and stromal cells in the homing and homeostasis of chronic lymphocytic leukemia B cells. Leuk Lymphoma. 2002:43(3):461-466.

17. Burger JA, Kipps TJ. CXCR4: a key receptor in the crosstalk between tumor cells and their microenvironment. Blood. 2006;107 (5):1761-1767.

18. Burger JA, Peled A. CXCR4 antagonists: targeting the microenvironment in leukemia and other cancers. Leukemia. 2009;23(1):43-52.
19. Liang Z, Brooks J, Willard $M$, et al. CXCR4/CXCL12 axis promotes VEGFmediated tumor angiogenesis through Akt signaling pathway. Biochem Biophys Res Commun. 2007;359(3):716-722.

20. Ping YF, Yao XH, Jiang JY, et al. The chemokine CXCL12 and its receptor CXCR4 promote glioma stem cell-mediated VEGF production and tumour angiogenesis via PI3K/AKT signalling. J Pathol. 2011;224(3):344-354

21. Sun X, Charbonneau C, Wei L, Yang W, Chen Q, Terek RM. CXCR4-targeted therapy inhibits VEGF expression and chondrosarcoma angiogenesis and metastasis. Mol Cancer Ther. 2013;12(7):1163-1170.

22. Cho BS, Kim HJ, Konopleva M. Targeting the CXCL12/CXCR4 axis in acute myeloid leukemia: from bench to bedside. Korean J Intern Med. 2017:32(2):248-257.

23. Kast RE. Profound blockage of CXCR4 signaling at multiple points using the synergy between plerixafor, mirtazapine, and clotrimazole as a new glioblastoma treatment adjunct. Turk Neurosurg. 2010;20(4):425429.

24. Konopleva MY, Jordan CT. Leukemia stem cells and microenvironment: biology and therapeutic targeting. J Clin Oncol. 2011;29(5):591-599.

25. Liu T, Li X, You S, Bhuyan SS, Dong L. Effectiveness of AMD3100 in treatment of leukemia and solid tumors: from original discovery to use in current clinical practice. Exp Hematol Oncol. 2016;5(1):19.

26. Redjal N, Chan JA, Segal RA, Kung AL. CXCR4 inhibition synergizes with cytotoxic chemotherapy in gliomas. Clin Cancer Res. 2006;12(22):6765-6771.

27. Uy GL, Rettig MP, Motabi IH, et al. A phase $1 / 2$ study of chemosensitization with the CXCR4 antagonist plerixafor in relapsed or refractory acute myeloid leukemia. Blood. 2012;119(17):3917-3924.

28. Balsas P, Esteve-Arenys A, Roldan J et al. Activity of the novel BCR kinase inhibitor IOS019 in preclinical models of B-cell nonHodgkin lymphoma. J Hematol Oncol. 2017;10(1):80

29. Kawano Y, Kobune M, Yamaguchi M, et al Ex vivo expansion of human umbilical cord hematopoietic progenitor cells using a coculture system with human telomerase catalytic subunit (hTERT)-transfected human stromal cells. Blood. 2003;101(2): 532-540.

30. Bosch R, Dieguez-Gonzalez R, Cespedes $M V$, et al. A novel inhibitor of focal adhesion signaling induces caspase-independent cell death in diffuse large B-cell lymphoma. Blood. 2011;118(16):4411-4420

31. Cardesa-Salzmann TM, Colomo L Gutierrez G, et al. High microvessel density determines a poor outcome in patients with diffuse large B-cell lymphoma treated with rituximab plus chemotherapy. Haematologica. 2011;96(7):996-1001

32. Moros A, Rodriguez V, Saborit-Villarroya I, et al. Synergistic antitumor activity of lenalidomide with the BET bromodomain inhibitor CPI203 in bortezomib-resistant mantle cell lymphoma Leukemia. 2014;28(10):2049-2059

33. Perry AM, Cardesa-Salzmann TM, Meyer PN, et al. A new biologic prognostic model based on immunohistochemistry predicts survival in patients with diffuse large B-cell lymphoma. Blood. 2012;120(11):22902296

34. Pérez-Nueno VI, Ritchie DW, Rabal O, Pascual R, Borrell JI, Teixidó J. Comparison of ligand-based and receptor-based virtual screening of HIV entry inhibitors for the CXCR4 and CCR5 receptors using 3D ligand shape matching and ligand-recepto docking. J Chem Inf Model. 2008;48(3): 509-533.

35. Ros-Blanco L, Anido J, Bosser R, et al. Noncyclam tetraamines inhibit CXC chemokine receptor type 4 and target glioma-initiating cells. J Med Chem. 2012;55(17):7560-7570

36. Reinholdt L, Laursen MB, Schmitz A, et al. The CXCR4 antagonist plerixafor enhances the effect of rituximab in diffuse large B-cell lymphoma cell lines. Biomark Res. 2016;4:12.

37. Campo E. MYC in DLBCL: partners matter Blood. 2015;126(22):2439-2440.

38. An J, Yang DY, Xu QZ, et al. DNA-dependent protein kinase catalytic subunit modulates the stability of c-Myc oncoprotein Mol Cancer. 2008;7:32.

39. Sears R, Nuckolls F, Haura E, Taya Y, Tamai K, Nevins JR. Multiple Ras-dependent phosphorylation pathways regulate Myc protein stability. Genes Dev. 2000;14(19):2501-2514

40. Banerjee C, Archin N, Michaels D, et al BET bromodomain inhibition as a nove strategy for reactivation of HIV-1. J Leukoc Biol. 2012;92(6):1147-1154

41. Chen Y, Jacamo R, Konopleva M, Garzon R, Croce C, Andreeff M. CXCR4 downregulation of let-7a drives chemoresistance in acute myeloid leukemia. J Clin Invest. 2013:123(6):2395-2407

42. Zeng Z, Shi YX, Samudio IJ, et al. Targeting the leukemia microenvironment by CXCR4 inhibition overcomes resistance to kinase inhibitors and chemotherapy in AML. Blood. 2009;113(24):6215-6224.

43. Chen J, Xu-Monette ZY, Deng L, et al. Dysregulated CXCR4 expression promote lymphoma cell survival and independently predicts disease progression in germinal center B-cell-like diffuse large B-cell lymphoma. Oncotarget. 2015;6(8):5597-5614

44. Bachelder RE, Wendt MA, Mercurio AM Vascular endothelial growth factor promotes breast carcinoma invasion in an autocrine manner by regulating the chemokine receptor CXCR4. Cancer Res. 2002;62(24):7203-7206

45. Tseng D, Vasquez-Medrano DA, Brown JM. Targeting SDF-1/CXCR4 to inhibit tumour vasculature for treatment of glioblastomas. Br J Cancer. 2011;104(12): 1805-1809.

46. Zhang Y, Zhao H, Zhao D, et al. SDF1/CXCR4 axis in myelodysplastic syndromes: correlation with angiogenesis and apoptosis. Leuk Res. 2012;36(3):281-286.

47. Kioi M, Vogel H, Schultz G, Hoffman RM, Harsh GR, Brown JM. Inhibition of vasculogenesis, but not angiogenesis, prevents the recurrence of glioblastoma after irradiation in mice. J Clin Invest. 2010;120(3):694 705.

48. Juarez J, Dela PA, Baraz R, et al. CXCR4 antagonists mobilize childhood acute lymphoblastic leukemia cells into the peripheral blood and inhibit engraftment. Leukemia. 2007;21(6):1249-1257.

49. Nervi B, Ramirez P, Rettig MP, et al. Chemosensitization of acute myeloid leukemia (AML) following mobilization by the CXCR4 antagonist AMD3100. Blood. 2009:113(24):6206-6214.

50. Hu Y, Gale M, Shields J, et al. Enhancement of the anti-tumor activity of therapeutic monoclonal antibodies by CXCR4 antagonists. Leuk Lymphoma. 2012;53(1):130-138. 
51. Stamatopoulos B, Meuleman N, De BC, et al. AMD3100 disrupts the cross-talk between chronic lymphocytic leukemia cells and a mesenchymal stromal or nurselike cell-based microenvironment: pre-clinical evidence for its association with chronic lymphocytic leukemia treatments. Haematologica. 2012;97(4):608-615

52. Pettersson S, Perez-Nueno VI, Ros-Blanco $\mathrm{L}$, et al. Discovery of novel non-cyclam polynitrogenated CXCR4 coreceptor inhibitors. ChemMedChem. 2008;3(10): 1549-1557.

53. Kashyap MK, Amaya-Chanaga CI, Kumar $\mathrm{D}$, et al. Targeting the CXCR4 pathway using a novel anti-CXCR4 IgG1 antibody (PF-06747143) in chronic lymphocytic leukemia. J Hematol Oncol. 2017;10(1):112.

54. Valera A, López-Guillermo A, CardesaSalzmann T, et al. MYC protein expression and genetic alterations have prognostic impact in patients with diffuse large B-cell lymphoma treated with immunochemotherapy. Haematologica. 2013;98(10):
1554-1562.

55. Hatano K, Yamaguchi S, Nimura K, et al. Residual prostate cancer cells after docetaxel therapy increase the tumorigenic potential via constitutive signaling of CXCR4 ERK1/2 and c-Myc. Mol Cancer Res. 2013;11(9):1088-1100.

56. Filippakopoulos P, Knapp S. Targeting bromodomains: epigenetic readers of lysine acetylation. Nat Rev Drug Discov. 2014;13(5):337-356.

57. Diaz T, Rodriguez V, Lozano E, et al. The BET bromodomain inhibitor CPI203 improves lenalidomide and dexamethasone activity in in vitro and in vivo models of multiple myeloma by blockade of Ikaros and MYC signaling. Haematologica. 2017;102(10):1776-1784

58. Boi M, Gaudio E, Bonetti P, et al. The BET bromodomain inhibitor OTX015 affects pathogenetic pathways in preclinical B-cell tumor models and synergizes with targeted drugs. Clin Cancer Res. 2015;21(7):16281638
59. Ceribelli M, Kelly PN, Shaffer AL, et al. Blockade of oncogenic IkappaB kinase activity in diffuse large B-cell lymphoma by bromodomain and extraterminal domain protein inhibitors. Proc Natl Acad Sci U S A. 2014;111(31):11365-11370.

60. Esteve-Arenys A, Valero JG, ChamorroJorganes A, et al. The BET bromodomain inhibitor CPI203 overcomes resistance to ABT-199 (venetoclax) by downregulation of BFL-1/A1 in in vitro and in vivo models of MYC+/BCL2+ double hit lymphoma. Oncogene. 2018;37(14):1830-1844

61. Chapuy B, McKeown MR, Lin CY, et al Discovery and characterization of superenhancer-associated dependencies in diffuse large B cell lymphoma. Cancer Cell 2013;24(6):777-790

62. Moros A, Bustany S, Cahu J, et al. Antitumoral activity of lenalidomide in in vitro and in vivo models of mantle cell lymphoma involves the destabilization of cyclin D1/p27KIP1 complexes. Clin Cancer Res. 2014;20(2):393-403. 